\title{
Study on the Shear Deformation Property of Warp-knit Fabrics
}

\author{
Part 1: Theory
}

\author{
By Yoshiki Yanagawa* and Sueo Kawabata**, Members, TMSJ \\ *Department of Textile Technology, Kyoto University of Industrial Arts and Textile Fibers, Kyoto \\ **Department of Polymer Chemistry, Kyoto University, Kyoto \\ Based on Journal of the Textile Machinery Society of Japan, Transactions, Vol. 26, No. 2, T23-33 (1973)
}

\section{Abstract}

The shear deformation property of two-bar tricot fabrics is analyzed, and the theory for calculating this property is dereloped by using a structural model. A unit structure of the model is constructed of three arcs of front-bar yarn and three arcs of back-bar yarn, and these arcs are stretched under shear deformation. This theory covers the general shear deformation of fabrics, for example, simple shear deformation, shear deformation under biaxial tensile deformation.

In this theory, the forces for stretching the fabric 3 are calculated from the forces required for straightening the arcs, while the frictional effect between yarns is taken into consideration, but the elongation and the compressive properties of yarn are out of consideration. The structure constants required for calcualation can be obtained easily from wale density, course density, fabric weight and yarn count.

KEY WORDS: WaRp KnitTed Fabrics, Bending Rigidity, Yarn to Yarn Friction

\section{Introduction}

Recently, the shear-deformation property of woven fabrics has been studied extensively and been made clear by some researchers as Grosberg[1], Niwa and Kawabata $[2,3]$ et. al. On the other hand, the shear property of knitted fabrics, whether it is of weft knitted fabrics or of warp knitted fabrics, remains unstudied owing to their complicated structure.

In this paper, a structure model of warp-knitted fabrics of two-bar tricot is presented and the shear-deformation property of the fabrics is analysed to calculate it from yarn properties.

The unit structure of the model is constructed of three arcs for front-bar yarn and three arcs for back-bar yarn. During shear deformation of the fabrics, the curvature of each arc is changed and the slippage of yarn at the cross over point occurs. These phenomena are treated in this theory. However the elongation and the compressive properties of the yarn are out of consideration.

\section{Theory}

Let us consider the general shear deformation, where shear and tensile deformations are applied simultaneously.

Let us take $\mathrm{X}_{1}$-axis in wale direction and $\mathrm{X}_{2}$-axis in course direction when there is no shear deformation, and put $\lambda_{1}$ as the stretch ratio of the fabric along $X_{1}$-axis and $\lambda_{2}$ as that along $X_{2}$-axis, respectively. The deformation is divided into two steps as following. First, the tensile deformation is applied along $X_{1}$ and $X_{2}$-axis by stretch ratios $\lambda_{1}$ and $\lambda_{2}$, and secondary the shear deformation is applied by shear angle $\psi$ as shown in Fig. 1. The stretch ratios along wale and course directions are then changed to $\lambda_{1}^{\prime}$ and $\lambda_{2}^{\prime}$ from $\lambda_{1}$ and $\lambda_{2}$ respectively by this second step.

There are two modes in the shear deformation as illustrated in Fig. 1, that is, the shear along $X_{1}$ axis, and the shear along $X_{2}$ axis. But these two modes are essentially

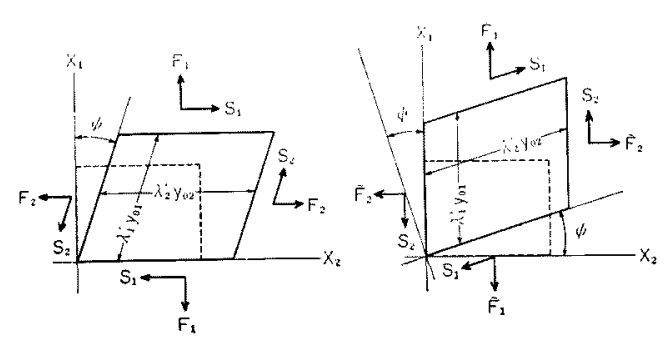

Fig. 1 Shear deformation under forced biaxial-extension 


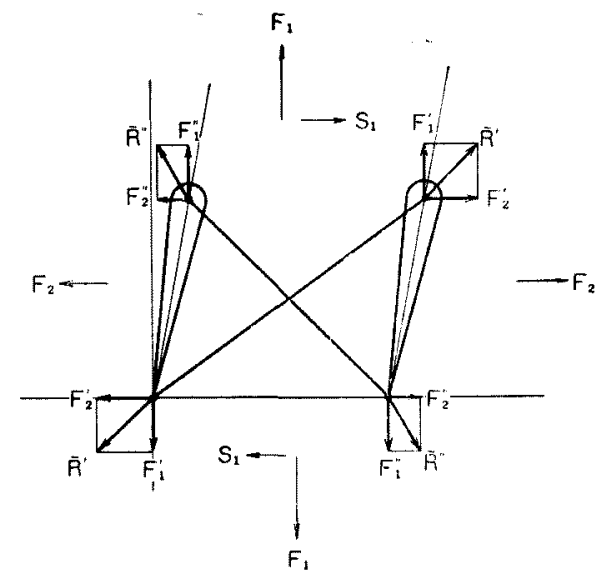

Fig. 2 External forces applied on a unit structure

the same to each other, and represented by $\lambda_{1}^{\prime}$ and $\lambda_{2}^{\prime}$. For this reason, only the shear deformation along $X_{2}$-axis is dealt with here.

Let $\tilde{R}^{\prime}$ and $\tilde{R}^{\prime \prime}$ be the external forces acting on the crossover points, and the components of $\tilde{R}^{\prime}$ and $\tilde{R}^{\prime \prime}$ in $\mathrm{X}_{1}$-axis are expressed by $F_{1}^{\prime}$ and $F_{1}^{\prime \prime}$, and those in $\mathrm{X}_{2}$-axis by $F_{2}^{\prime}$ and $F_{2}{ }^{\prime \prime}$. Put $F_{1}$ be the tensile force acting on fabric along $\mathrm{X}_{1}$-axis per wale (g/wale), and $S_{1}$ be shear force per wale (g/wale). As shown in Fig. 2, forces $F_{1}^{\prime}$ and $F^{\prime \prime}{ }_{1}$ are components of $F_{1}$, and $F_{2}^{\prime}$ and $F_{2}^{\prime \prime}$ are of $F_{2}$.

In the case of a plain tricot-fabric, the relations between these forces are as follows.

$$
\begin{aligned}
& S_{1}=F_{2}{ }^{\prime}-F_{2}{ }^{\prime \prime} \\
& F_{1}=F_{1}{ }^{\prime}+F_{1}{ }^{\prime} \\
& F_{2}=F_{2}^{\prime}+F_{2}^{\prime \prime}
\end{aligned}
$$

\subsection{Unit Stuctural Model}

The unit structural model used here before the deformation cccurs is the same as that in the biaxial tensile theory. ${ }^{[4,5]}$ That is, the model is composed of a needle loop having two arcs, a sinker loop having an arc and of the crossover region, for each of the front-bar yarn and the back-bar yarn, respectively, as illustrated in Fig. 3. It is assumed that the planes which contain each arc are parallel to $\mathrm{X}_{2}$-axis.

Structural constants are expressed as follows: Let $y_{01}$ and $y_{02}$ be the course spacing and the wale spacing before the deformation occurs. Let $D$ be the yarn diameter and $n$ the number of wales crossed by underlapping. Then, the arc length of sinker loop, $L_{a 1 f}$, and the arc lengths of needle loop, $L_{a 2 f}$ and $L_{a 3 f}$ in the front-bar yarn, and those lengths $L_{a 1 b}, L_{a 2 b}$ and $L_{a 3 b}$ in the back-bar yarn, are calculated from the equations ${ }^{[4,5]}$ as follows, where suffix 1 refers to the sinker loops 2 and 3, to the inside arc and the outside arc of the needle loop, as shown in Fig. 3.

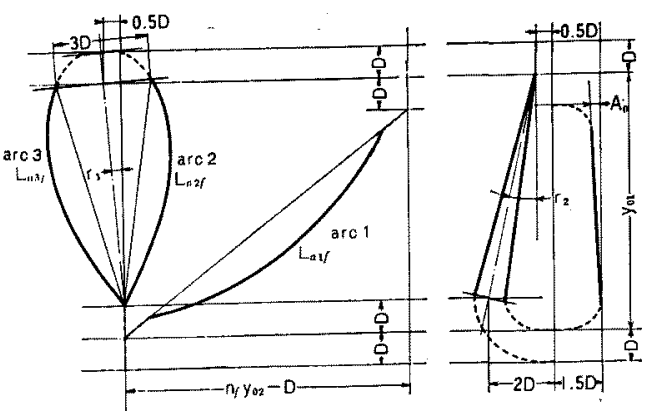

Fig. 3 Unit structural model (for front-bar yarn)

$$
\begin{aligned}
& \left.\begin{array}{l}
L_{a 1 f}=\sqrt{\left(\lambda_{c} n_{f} y_{02}-D\right)^{2}+\left(\lambda_{c} y_{01}-D\right)^{2}+\overline{A_{c}^{2}}}-2 D \\
L_{a 1 t^{\prime}}=\sqrt{\left(\lambda_{c} n_{1} y_{02}-D\right)^{2}-\left(\lambda_{c} y_{01}-D\right)^{2}+\bar{A}_{c}^{2}}-2 D
\end{array}\right\} \\
& L_{1 ; 2,}=L_{a 13 r}=\sqrt{\left(\lambda y_{01}-D\right)^{2}+5 \overline{J^{2}}} \\
& \left.L_{t a 2 b}=L_{u 3 b}=\sqrt{\left(\lambda r_{1} y_{01}-D\right)^{2}+9 J^{2}}\right\} \\
& A_{e}=2.5 D^{2} /\left(\lambda_{c} y_{01}-D\right)
\end{aligned}
$$

where $\lambda_{c}$ is the critical tensile ratio in a biaxially equal extension and can be obtained from the previous tensile theory ${ }^{[4,5]}$. Suffixes $f$ and $b$ refer to the front-bar yarn and the back-bar yarn respectively.

The distance between two ends of a sinker arc, $d_{01}$, that of $\operatorname{arc} 2, d_{02}$, and that of arc $3, d_{03}$, are geometrically calculated by

$$
\begin{aligned}
& d_{01}=\sqrt{\left(n y_{02}-D\right)^{2}+\left(y_{01}-D\right)^{2}+A_{0}{ }^{2}-2 D} \\
& \left.d_{011}=\sqrt{ }\left(n y_{02}-D\right)^{2}+\left(y_{01}-D\right)^{2}+A_{0}{ }^{2}-2 D\right) \\
& \text { … (5) } \\
& I_{02,}=d_{03}=\sqrt{\left.\left(y_{01}-D\right)^{2}+5\right)^{2}} \\
& d^{\prime} 0_{2 t}=d_{03^{\prime}}=\sqrt{\left(y_{01}-J\right)^{2}+9 D^{2}} \\
& \left.A_{0}=2.5 I\right)^{2} /\left(y_{01}-D\right)
\end{aligned}
$$

Thus, the shapes of all loops and the unit structure with no deformation are defined.

It is assumed that, during shear deformation, arcs in the unit structure are stretched by two external forces $P$ and $Q$ as a built-in beam, as shwon in Fig. 4, while the crossover region does not deform. Accordingly, tensile force $F$ and shear force $S$ are obtained from the total sum of the components of forces required for straightening six arcs, namely three arcs of the front-bar yarn and three arcs of the back-bar yarn. In this paper, the elongation and the compressive properties of yarn are out of consideration.

For introducing the tensile and compressive properties of yarn in the theory, Kawabata's method will be used, by which a deformed process is analyzed by dividing it into two regions, that is, the bending region and the stretching region $^{[6]}$. Following this division, this theory treats the

$$
\text { Journal of The Textile Machinery Society of Japan }
$$




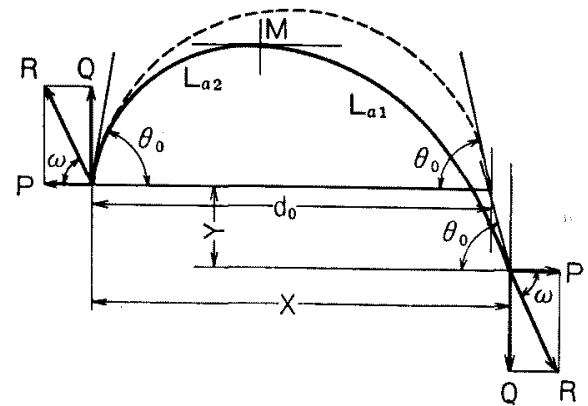

Fig. 4 Deformation of an elastic bar with built-in ends

bending region. The compressive property of yarn is introduced in the stretching region, which will be described in the following paper.

2.2 Deformation of elastic-arc rods

The stretching behavior of an elastic-thin-arc rod, one end of which is fixed and the other stretched by $\Delta X(=X-$ $d_{0}$ ) toward the chord direction and by $Y$ toward vertical to the chord, as shown in Fig. 4, is analyzed here.

Let $\kappa_{0}$ be its curvature, $d_{0}$ the distance between two ends of the arc with no deformation, and $\theta_{0}$ the fixed angle at each end of the arc.

When forces act on both ends, the arc is deformed as shown in a solid line in Fig. 4. This deformation can be analyzed by dividing the rod into two parts, each length of which is $L_{a 1}$ and $L_{a 2}$ respectively. Point $M$ in the figure is the border of the two parts, and there the tangent of the curved rod is parallel to $\mathrm{X}$-axis. This deformation is considered to be such that one end of the arc is subjected with two forces $P$ and $Q$ while keeping $\theta_{0}$ fixed, and the other end of the point $M$ is fixed in two parts of the lengths $L_{1 a}$ and $L_{a 2}$ respectively, as shown Fig. 5 .

Taking the coordinate axes as shown in the figure, the differential equation of the deffection curve in each part is given by

$$
\begin{aligned}
& B\left(\frac{d \theta_{1}}{d S_{1}}-\kappa_{0}\right)+M_{01}-P_{y_{1}}+Q x_{1}=0 \\
& B\left(\frac{d O_{2}}{d S_{2}}-\dot{\kappa}_{0}\right)+M_{02}-P y_{2}-Q x_{2}=0
\end{aligned}
$$

where

$B$ : bending rigidity of the rod

$S$ : arc length from the origin

$\theta$ : tangential angle

$M_{0}$ : bending moment at the origin,

and suffixes 1 and 2 refer to the two divided parts, $L_{a 1}$ and $L_{a 2}$ respectively.

At point $M$, the following equation is obtained.

$$
-P^{\prime} Y_{1}+Q X_{1}+M_{01}=-I^{\prime} Y_{2}-Q X_{2}+M_{02} \cdots \cdots(10)
$$

Vol. 20 No. 4 (1974)

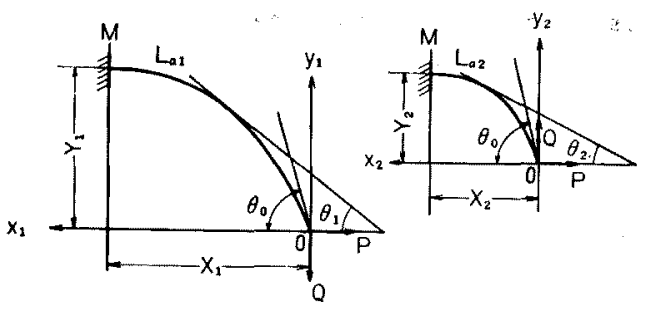

(a)

(b)

Fig. 5 Dividing the arc of Fig. 4 into two parts

$$
\begin{aligned}
& L_{1,1}+L_{u(2}=L_{t \mid} \\
& X_{1}+X_{2}=X \cdots \\
& Y_{1}-Y_{2}=Y \cdots
\end{aligned}
$$

In some cases, a point of inflexion appears on the half side (part 1) of the arc.

A The case with no point of inflexion.

Substituting the following into eq. (8)

$$
\left.\begin{array}{l}
a=P / B \\
b=Q / B \\
C_{1}=K_{0}-M_{01} / B
\end{array}\right\}
$$

and differentiating with respect to $S$, we obtain

$$
\frac{\left.d^{2} /\right)_{1}}{d S_{1}^{2}}=u \sin \left(\theta_{1}-(i)\right)
$$

where

$$
\begin{aligned}
& u=\sqrt{a^{2}+b^{2}}=\sqrt{P^{2}+Q^{2}} / B=R / B \\
& \left(b=\tan ^{-1}(l / a)=\tan ^{-1}(Q / P) \cdots \ldots \ldots\right.
\end{aligned}
$$

Putting $d \theta_{1} / d s_{1}=Z_{1}$, and integrating eq. (15), when $\theta_{1}=\theta_{0}$ and $Z_{1}=C_{1}$, we have

$$
\begin{aligned}
\frac{d \theta_{1}}{d S_{1}} & =-\sqrt{C_{1}{ }^{2}+2 u \cos \left(\theta_{0}-\omega\right)-2 u \cos \left(\theta_{1}-\omega\right)} \\
& =-2 \sqrt{u} \sqrt{1 / K_{1}+\sin ^{2} \frac{\left(\theta_{1}-\omega\right)}{2}} \ldots \ldots \ldots .(18)
\end{aligned}
$$

where

$$
K_{1}=4 u /\left(C_{1}^{2}-4 u \sin ^{2} \frac{\left(\theta_{0}-\omega\right)}{2}\right)
$$

Then, we have

$$
\begin{array}{r}
I_{u 1}=\int d S_{1}=\frac{1}{2 \sqrt{u}} \int_{0}^{\theta_{0}} f_{1}\left(\theta_{1}\right) d \theta_{1} \ldots \ldots \ldots . .(20) \\
X_{1}=\int \cos \theta_{1} d S_{1}=\frac{1}{2 \sqrt{u}} \int_{0}^{\theta_{0}} f_{1}\left(\theta_{1}\right) \cos \theta_{1} d \theta_{1} \\
\ldots \ldots \ldots \ldots \\
Y_{1}=\int \sin \theta_{1} d S_{1}=\frac{1}{2 \sqrt{u}} \int_{0}^{\theta_{0}} f_{1}\left(\theta_{1}\right) \sin \theta_{1} d \theta_{1}
\end{array}
$$

where

$$
f_{1}\left(t_{1}\right)=1 / \sqrt{1 / K_{1}+\sin ^{2}\left(t_{1}-\omega\right)}
$$


In solvong eq. (9) similarly as before, we have the following equations,

$$
\begin{aligned}
& L a_{2}=\frac{1}{2} \int_{0}^{l_{0}} f_{2}\left(l_{2}\right) d 0_{2} \\
& x_{2}=\frac{1}{2 \sqrt{21}} \int_{0}^{n_{0}} f_{2}\left(t_{2}\right) \cos \theta_{2} d \theta_{2} \\
& r_{2}=\frac{1}{2 \sqrt{u}} \int_{0}^{\theta_{0}} f_{2}\left(t_{2}\right) \sin \theta_{2} d t_{2}
\end{aligned}
$$

where

$$
\begin{aligned}
& f_{2}\left(h_{2}\right)=1 / \sqrt{1 / K_{2}+\sin ^{2} \frac{\left(\theta_{2}+m\right)}{2}} \ldots \ldots \ldots \ldots \ldots(27) \\
& K_{2}=4 u /\left(C_{2}{ }^{2}-4 u \sin ^{2} \frac{\left(\theta_{0}+\omega\right)}{2}\right) \\
& C_{2}=\kappa_{0}-M_{02} / B
\end{aligned}
$$

Dividing both sides of eq. (10) by $u$ and $B$,

$$
\begin{aligned}
& -\cos \omega \cdot Y_{1}+\sin \left(\omega \cdot X_{1}+\frac{1}{*} \frac{M_{01}}{B}\right. \\
& =-\cos \omega \cdot Y_{2}-\sin \omega \cdot X_{2}+\frac{1}{"} M_{02}
\end{aligned}
$$

From eqs. (14) and (15), $M_{01} / B$ is given by

$$
\frac{M_{02}}{B}=\sqrt{u} \sqrt{4 / K_{1}+4 \sin _{2} \frac{\left(\theta_{0}-(0)\right.}{2}}+\kappa_{0} \cdots \cdots
$$

From eqs. (28) and (29), $M_{o 2} / B$ is also given by

$$
\frac{M_{03}}{B}=\sqrt{u} \sqrt{4 / K_{2}+4 \sin ^{2} \frac{\left(\sigma_{0}+\omega\right)}{2}}+\kappa_{0} \cdots \cdots(32)
$$

Substituting eqs. (21), (22), (25), (26), (31) and (32) into eq. (30), then the left side of the equation, say $V_{1}$, become

$$
\begin{aligned}
V_{1} & =-\frac{1}{2} \cos \omega \int_{0}^{\theta_{0}} f_{1}\left(\theta_{1}\right) \sin \theta_{1} d \theta_{1}+\frac{1}{2} \sin \omega x \\
& \int_{0}^{g_{0}} f_{1}\left(\theta_{1}\right) \cos \theta_{1} d \theta_{1} \\
& +\sqrt{4 / K_{1}+4 \sin ^{2} \frac{\left(\theta_{0}-\omega\right)}{2}} \ldots \ldots \ldots \ldots \ldots \ldots \ldots \ldots \ldots \ldots
\end{aligned}
$$

and the right side, say $V_{2}$, becomes

$$
\begin{aligned}
V_{2} & =-\frac{1}{2} \cos \omega \int_{0}^{\theta_{0}} f_{2}\left(\theta_{2}\right) \sin \theta_{2} d \theta_{2}-\frac{1}{2} \sin \omega \times \\
& \int_{0}^{\theta_{0}} f_{2}\left(t_{2}\right) \cos \theta_{2} d \theta_{2} \\
& +\sqrt{4 / K_{2}+4 \sin _{2}\left(\theta_{0}+\omega\right)} \ldots \ldots \ldots \ldots \ldots \ldots \ldots \ldots \ldots \ldots
\end{aligned}
$$

If $\omega$ is given, eq. (30) can be solved for $K_{1}$ and $K_{2}$. But it is very difficult to obtain the analytical solution for them. So the numerical method has been tried, and we found that $K_{1}$ and $K_{2}$ are equal for any set of $\theta_{0}$ and $\omega$.

Now we put $K_{1}=K_{2}=K$, and consider that $\theta_{0}, \omega$ and $K$ are parameters.

Substituting them into eqs. (35), (36) and (37), $2 \sqrt{u} L_{a}$ $2 \sqrt{u} X$ and $2 \sqrt{u} Y$ can be obtained by the numerical integration of the following equations.

$$
\begin{aligned}
& 2 \sqrt{u} L_{a}=\int_{0}^{\theta_{0}} f_{1}\left(\theta_{1}\right) d \theta_{1}+\int_{0}^{\theta_{0}} f_{2}\left(\theta_{2}\right) d \theta_{2}=J_{L}\left(u_{0} .\right. \\
& \text { (11, } \left.K^{*}\right) \\
& 2 \sqrt{2} x=\int_{0}^{\theta_{0}} f_{1}\left(H_{1}\right) \cos \theta_{1} d \|_{1}+\int_{0}^{\theta_{0}} f_{2}\left(\|_{2}\right) \cos x \\
& \theta_{2} d \theta_{2}=I_{x}\left(\theta_{0}, \omega, K\right) \\
& 2 \sqrt{\prime} Y=\int_{0}^{\mu_{0}} f_{1}\left(\theta_{1}\right) \sin \theta_{1} d \theta_{1}-\int_{0}^{\theta_{0}} f_{2}\left(\theta_{2}\right) \sin \theta_{2} \\
& d \prime_{2}=J_{Y}\left(\theta_{0},(\omega, K)\right.
\end{aligned}
$$

The method to calculate $P$ and $Q$ from these equations will be described later.

B The case with a point of inflexion

When the inflexion point appears in the half side of the arc as shown in Fig. 6, the rod deformation can be analyzed by separating it at the infection point $I$ into two parts, the lengths of which are $L_{a 1}^{\prime}$ and $L^{\prime \prime}{ }_{a 1}$. Even if a point of inflextion appears, eqs. (8), (9) and (10) are also applicable. In the part of length $L_{a 1}^{\prime}$, positive sign must be taken in eq. (18) since $d \theta_{1} / d s_{1}$ is positive, and

$$
\frac{d \prime_{1}}{d s_{1}}=2, u \sqrt{1 / K_{1}+\sin ^{2}\left(\frac{\left.(1)_{1}-\omega\right)}{2}\right)}
$$

This equation can also be expressed as following,

$$
\frac{d l_{1}}{d S_{1}^{\prime}}=2, " \| \sqrt{1 / K_{1}^{\prime}-\cos ^{2}\left(\theta_{1}-(\omega)\right.}
$$

where

$$
K_{1}^{\prime}-4 \mu /\left(c_{1}^{2}+4 \| \cos ^{2} \frac{\left(\theta_{0}-(b)\right.}{2}\right)
$$

The relation between $K_{1}$ and $K^{\prime}{ }_{1}$ is given by

$$
1 / K_{1}=1 / K_{1}^{\prime}-1
$$

Since $d \theta_{1} / d s_{1}=0$ at point $I$, the angle between the tangent at point $I$ and $X$-axis, is given by

$$
\theta_{1}=2 \cos ^{-1} \sqrt{K_{1}^{\prime}}+\cdots
$$

Putting $\sqrt{1 / K_{1}}=k^{\prime}{ }_{1}$, and $\cos \left(\theta_{1}-\omega\right) / 2=k^{\prime}{ }_{1} \cos \xi^{\prime}$, we have

$$
\begin{aligned}
& \sqrt{1 / K_{1}^{\prime}}=k_{1}^{\prime}, \cos \frac{\left.()_{1}-(m)\right)}{2}=k_{1}{ }^{\prime} \cos \xi^{\prime} \text {. }
\end{aligned}
$$

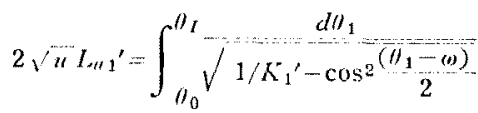

$$
\begin{aligned}
& =\int_{\Sigma_{0}^{\prime}}^{5 t^{\prime}} \frac{-2 d s^{\prime}}{\sqrt{1-k_{1}^{\prime 2} \cos ^{2} \xi^{\prime}}}
\end{aligned}
$$


where

$$
\begin{aligned}
& \xi_{0}^{\prime}=\cos ^{-1}\left(\cos ^{\left(\theta_{0}-(\theta)\right.} 2 / k_{1}^{\prime}\right) \\
& \xi_{I^{\prime}}=0
\end{aligned}
$$

So the following equation is obtained,

$$
\begin{aligned}
\cos \theta_{1} & =\left(2 k_{1}{ }^{\prime 2} \cos ^{2} \xi^{\prime}-1\right) \cos \left(n+2 k_{1}^{\prime} \times\right. \\
& \sqrt{1-k_{1}{ }^{\prime 2} \cos ^{2} \xi^{\prime}} \cdot \sin (1) \cdot \cos \xi^{\prime} \cdots \ldots \ldots \ldots \ldots
\end{aligned}
$$

Therefore we obtain

$$
\begin{aligned}
& 2 \sqrt{ } u x_{1}^{\prime}=\int_{\theta_{0}}^{\theta_{r}} \frac{\cos \theta_{1} d \theta_{1}}{\sqrt{1 / K_{1}-\cos ^{2}\left(\theta_{1}-\theta\right)}} \\
& =-2 \cos \omega \int_{50^{\prime}}^{5 t^{\prime}} \frac{2 k_{1}^{\prime 2} \cos ^{2} \xi^{\prime}-1}{1-k_{1}^{\prime 2} \cos ^{2} \xi^{\prime}} d \xi^{\prime}-4 k_{1}^{\prime} \sin \omega x \\
& \int_{50^{\prime}}^{5 \frac{5}{l^{\prime}}} \cos \xi^{\prime} d \xi^{\prime \prime}
\end{aligned}
$$

For calculating $Y_{1}^{\prime}$, we obtain the following in the same manner.

$$
\begin{aligned}
& 2 \sqrt{ } u Y_{1}^{\prime}=4 k_{1}{ }^{\prime} \cos \omega \int_{\xi 0^{\prime}}^{5 \iota^{\prime}} \cos z^{\prime} d \xi^{\prime}-2 \sin \omega x \\
& \int_{\xi 0^{\prime}}^{\xi 1^{\prime}} \frac{2 k_{1}^{\prime 2} \cos ^{2} \xi^{\prime}-1}{1-k_{1}^{\prime 2} \cos ^{2} \xi^{\prime}} d \xi
\end{aligned}
$$

Regarding the part of length $L^{\prime \prime}{ }_{a 1}$, putting the origin at point I and taking the coordinate axes, $x^{\prime \prime}$ and $y^{\prime \prime}$, as shown in Fig. 6, then eq. (8) is rewritten as following,

$$
\begin{aligned}
& B\left(\frac{d \theta_{1}}{d S_{1}}-\kappa_{0}\right)+M_{01}-P\left(Y_{1}^{\prime}+y_{1}^{\prime \prime}\right)+Q\left(X_{1}{ }^{\prime}+x_{1}{ }^{\prime \prime}\right) \\
& =0
\end{aligned}
$$

Since $x^{\prime \prime}=0, y^{\prime \prime}=0$ and $d \theta_{1} / d s_{1}=0$ at $\theta_{1}=\theta_{I}$, eq. (47) becomes

$$
\begin{array}{r}
\quad B x_{0}=M_{01}-P Y_{1}^{\prime}+O X_{1}{ }^{\prime} \\
\therefore \frac{d \theta_{1}}{d S_{1}}-a y_{1}^{\prime \prime}+b x_{1}^{\prime \prime}=0 \ldots .
\end{array}
$$

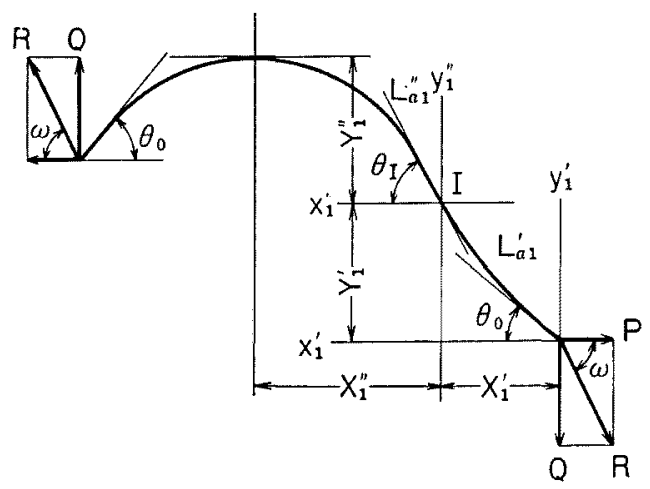

Fig. 6 Deforamation of an arc having an inflexion point

Vol. 20 No. $4(1974)$
This equation can be solved in the same manner as the case of $L_{a 1}^{\prime}$. Remenbering that $d \theta_{1} / d s_{1}$ is negative, we have

$$
\begin{aligned}
& \frac{d \prime_{1}}{d s_{1}}-\sqrt{2 i \cos \left(1 I_{1}-(1)\right)-2 i} \cos \left(\prime_{1}-(1)\right) \cdots(50)
\end{aligned}
$$

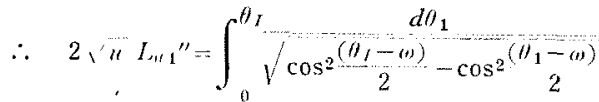

Putting $\cos \left(\theta_{1}-\omega\right) / 2=k^{\prime \prime}{ }_{1}$ and $\cos \left(\theta_{1}-\omega\right) / 2=k^{\prime \prime}{ }_{1} \cos \xi^{\prime \prime}$, we obtain

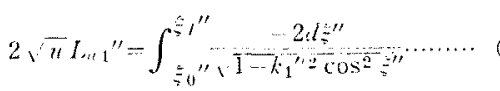

Here, the integral range must be taken between $\xi^{\prime \prime}{ }_{0}=\cos ^{-1}$ $\left(\cos (-\omega / 2) / k^{\prime \prime}{ }_{1}\right)$

$$
\text { and } \xi^{\prime \prime}{ }_{1}=0 \text {, }
$$

where $k_{1}^{\prime}=k^{\prime \prime}{ }_{1}$ because $k_{1}^{\prime}=\cos \left(\theta_{I}-\omega\right) / 2$ can be obtained from eq. (42).

Therefore, the value of $L^{\prime \prime}{ }_{a 1}$ can be calculated by replacing $\xi_{0}{ }^{\prime}$ by $\xi_{0}{ }^{\prime \prime}$ in eq. (43). $X^{\prime \prime}$ and $Y^{\prime \prime}$ can also be calculated in the similar way. Then, by using the following relations,

$$
\begin{aligned}
& X_{t 11}=L_{* 11}{ }^{\prime}+L_{n+1}{ }^{\prime \prime} \\
& X_{1}=X_{1}^{\prime}+X_{1}^{\prime \prime} \\
& Y_{1}=Y_{1}^{\prime}+Y_{1}^{\prime \prime}
\end{aligned}
$$

the relation between $K_{1}$ and $K_{2}$ is determined, similarly to the case with no point of inflexion. This has led to find that $K_{1}=K_{2}$. By substituting these values into eqs. (11), (12) and (13), $2 \sqrt{u} L_{a}, 2 \sqrt{u} X$ and $2 \sqrt{u} Y$ can be obtained.

The condition to have a point inflexion is given by $\theta_{0}<\omega$ and , (54)

$$
K_{1}=-1 / \sin 2 \frac{\left.(1)_{0}-(1)\right)}{2} \text {. }
$$

The dependency of the point of inflexion on $K_{1}$ is shown in Table 1.

Table 1 Range of $K_{1}$ to have an inflexion point

\begin{tabular}{cccc}
\hline & & \multicolumn{2}{c}{ Inflection point } \\
\cline { 3 - 4 }$K_{1}$ & $\theta_{0}$ & None & Exists \\
\cline { 3 - 3 }$K_{1} \quad \theta_{0} \geqq w$ & $0 \sim+\infty$ & None \\
& & $0 \sim+\infty$ & \\
& $\theta_{0}<w$ & $-\infty \sim-\frac{1}{\sin ^{2} \frac{\left(\theta^{0}-w\right)}{2}}-\frac{1}{\sin ^{2} \frac{\left(\theta_{0}-w\right)}{2}} \sim-0$
\end{tabular}

C. Calculation of forces $P$ and $Q$

For calculating $P$ and $Q$, forces acting on both ends of an arc, $K$ and $\omega$ must be determined by solving eqs. (35), (36) and (37) for given values of $X, Y$ and $L_{a}$. 


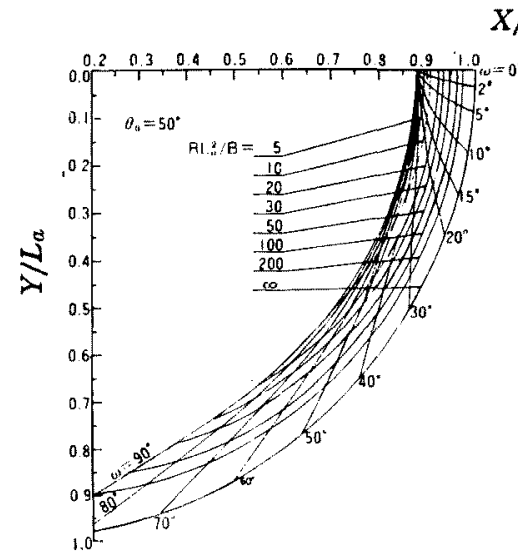

(a) $\theta_{0}=50^{\circ}$
$X / L_{a}$

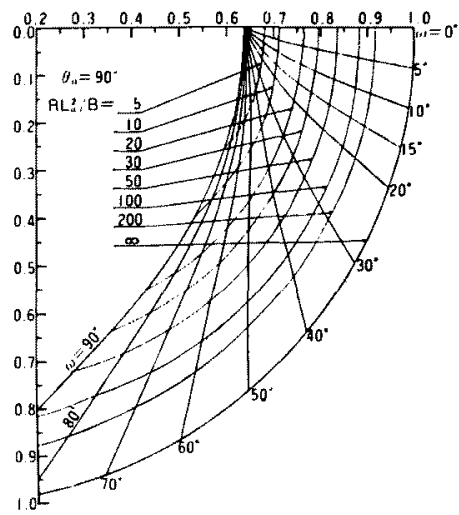

(b) $\theta_{0}=90^{\circ}$

Fig. 7 Relation among $X / L_{a}, Y / L_{a}$ and $R L^{2}{ }_{a} / B$

Eqs. (35), (36), and (37) can be rewritten as

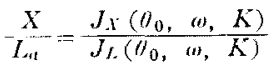

$$
\begin{aligned}
& \frac{Y}{L_{\alpha}}=\frac{J_{Y}\left(\eta_{0}, \omega, K\right)}{J_{L}\left(0_{0}, \omega, K\right)}
\end{aligned}
$$

When $X / L_{a}$ and $Y / L_{a}$ are given, values of $K$ and $\omega$ which satisfy the above eqs. (55) and (56) simultaneously can be solved by a graphical method instead of an analytical method to evade the calculating difficulty. As an example, in Fig. 7 are shown the contour lines of $\omega$ and $[R]$ defined by

$$
[R]=R L_{a}{ }^{2} / B \text {. }
$$

Thus, the values of $[R]$ and $\omega$ corresponding to $X / L_{a}$ and $Y / L_{a}$ can be found directly from this graph. $P$ and $Q$ are, then, calaulated by the following equations.

$$
\begin{aligned}
P & =\frac{[R] \cdot B}{L_{\alpha}{ }^{2}} \sqrt{1+\tan ^{2}(\omega)} \\
Q & =P \tan (\theta \ldots \ldots \ldots \ldots
\end{aligned}
$$

2.3 Shear deformation property of a unit structure

When the displacement of each arc-end in a unit structure is given, the tensile forces acting upon the ends are calculated by applying the deformation theory of an elastic bar above-mentioned.

When the fabric is deformed with shear angle $\phi$ and with stretch ratios $\lambda_{1}$ and $\lambda_{2}$, the plane formed by the arc in a unit structure tilts from $O X_{2} X_{03} X_{13}$ to $O X^{\prime}{ }_{2} X^{\prime}{ }_{03} X^{\prime}{ }_{13}$ as shown in Fig. 8. So, the tensile theory of a bar above mentioned, which treats two dimensions, cannot be applied strictly. Since the slant angle, $\alpha\left(=\tan ^{-1}\left(\overline{O X}_{3} / \overline{O X}_{1}\right)\right.$, of the plane is usually very small, and only such a case is dealt with here as the displacement $\Delta X_{1}$ is small, it is permitted to consider that the arc is subjected to two dimentsional

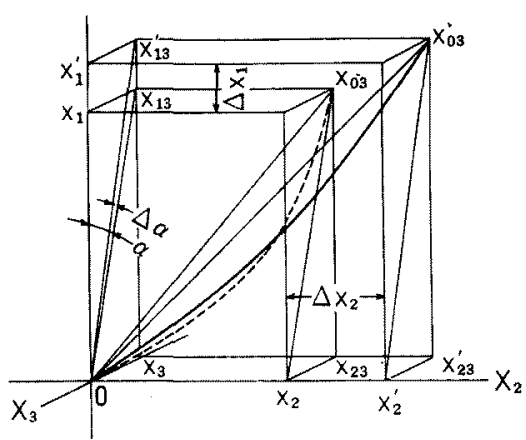

Fig. 8 Deformation of an arc in a unit structure

deformation in a plane $O X_{2}^{\prime} X^{\prime}{ }_{03} X^{\prime}{ }_{13}$. Thus, the arc can be assumed to be stretched by $\Delta X_{2}$ in the direction of $\mathrm{X}_{2}$-axis and by $\left[\Delta X_{1}\right]$ in the direction perpendicular to $X_{2}$-axis in plane $O X^{\prime}{ }_{2} X^{\prime}{ }_{03} X^{\prime}{ }_{13}$, where $\left[\Delta X_{1}\right]$ is given by

$$
\left[J X_{1}\right]=\sqrt{\left(O X_{1}+J X_{1}\right)^{2}+O X_{3}{ }^{2}}-\sqrt{O X_{1}{ }^{2}+X O_{3}{ }^{2}}
$$

So, the displacement of each arc in a unit structure is as follows:

When $\psi=0, \Delta X_{1}$ takes the same value with all arcs, and $\Delta X_{1}=\left(\lambda_{1}-1\right) y_{01}$.

For sinker loops, $\overline{O X_{1}}, \overline{O X_{2}}, \overline{O X_{3}}$ and $\Delta X_{2}$, we have

$\Delta X_{1}=\left(\lambda_{1}-1\right) y_{01}$

$$
\begin{aligned}
& \overline{O X_{1}}=y_{01}-D\left\{1+2\left(y_{01}-D\right) /\left(d_{01}+2 D\right)\right\}, \\
& O X_{2}=y_{02}-D\left\{1+2\left(n y_{02}-D\right) /\left(d_{01}+2 D\right)\right\} \\
& \Delta X_{2}=\left(\lambda_{2}-1\right) n \cdot y_{02}, \quad \overline{O X_{3}}=A_{0}
\end{aligned}
$$

Journal of The Textile Machinery Society of Japan 


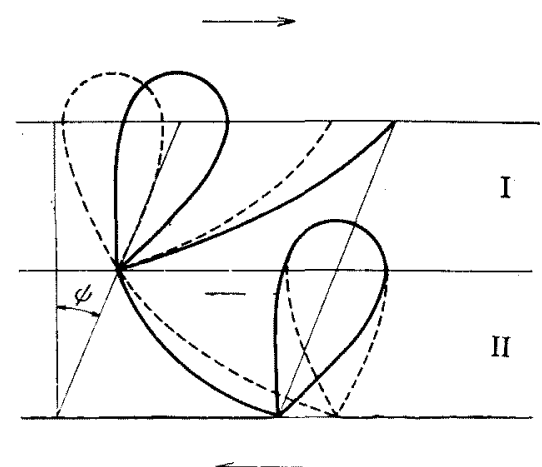

Fig. 9 Shear deformation of a structure, composed of type I and II

For needle loops,

$$
\begin{aligned}
\Delta X_{2} & =0, \quad \overline{O X_{1}}=y_{01}-D \\
\overline{O X_{2}} & =D \quad(\text { for } \operatorname{arcs}, 2 f \text { and } 2 b) \\
& =2 D \quad(\text { for } \operatorname{arcs}, 3 f \text { and } 3 b) \\
\overline{O X_{3}} & =D \quad(\text { for } \operatorname{arc} 3 f) \\
& =2 D \quad(\text { for } \operatorname{arcs}, 2 f \text { and } 3 b) \\
& =3 D \quad(\text { for } \operatorname{arc} 2 b) .
\end{aligned}
$$

When the fabric is subjected to the deformation of shear angle $\phi, \Delta X_{2}$ is increased or decreased by $\tan \psi \times \lambda_{1} y_{01}$, depending upon the position of the loop as described in the following.

As shown in Fig. 9, when the front-bar yarn is subjected to deformation of type $I$, the back-bar yarn must take type II. In the case of type I, $\Delta X_{2}$ must increase for arcs 1 and 2 , and must decrease for arc 3 . In the case of type II, $\Delta X_{2}$ must decrease for $\operatorname{arcs} 1$ and 2 , and must increase for $\operatorname{arc} 3$.

The displacements $X$ and $Y$, defined before, are expressed by $\left[\Delta X_{1}\right]$ and $\Delta X_{2}$ as following.

$$
\left.\begin{array}{l}
X=\left\{\Delta X_{1}\right] \sin \varphi_{0}+\Delta X_{2} \cdot \cos \varphi_{0}+d_{0} \\
Y=\left\{\Delta X_{1}\right] \cos \varphi_{0}-\Delta X_{2} \sin \varphi_{0}
\end{array}\right\} \cdots(60)
$$

where $\phi_{0}$ is given by

$$
\varphi_{0}=\cos ^{-1}\left(\overline{O X_{2}} /\left(d_{0}+\delta\right)\right)
$$

and $\delta$ is $2 D$ for a sinker loop and zero for a needle loop.

The angle of the fixed arc end, $\theta_{0}$, is obtained by

$$
d_{0} / L_{a}=\theta_{0} / \sin \theta_{0}
$$

where $L_{a}$ is the arc length, $d_{o}$ the distance between both ends of the arc, and these values are calculated by eqs. from (2) to (7). Ther fore, from $X$ and $Y$ obtained, $P$ and $Q$ (or $R$ and $\omega$ ) are calculable by solving eqs. (55) to (58) or graphically as described before.

2.4 Effect of frictional property of yarn

A yarn contact at crossover region

In the actual deformation of the fabric, however, yarns

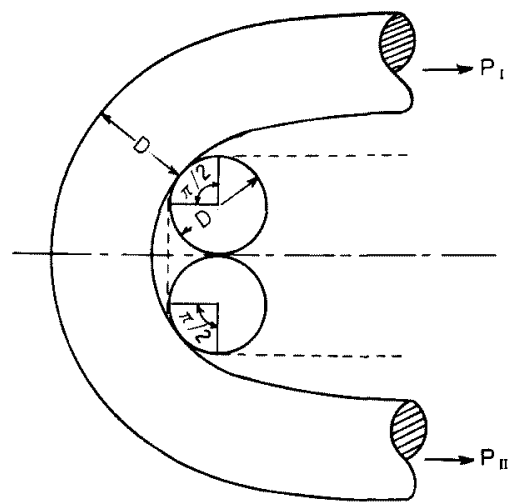

Fig. 10 Shape of yarn configuration in crossover region

are not fixed at the crossover point. In the structural model in this paper, it is assumed that the crossover region has the structure as shown in Fig. 10. If yarns are perfectly flexible, the total contact angle in the crossover region, $\Phi$, is $\pi$ as shown in a dotted line in that figure. But in the actural structure, the contact angle will be less than $\pi$, and point contact may arise in many cases, because of yarn rigidity.

If it is assumed that $\Phi=\pi$, thecalculation method in this paper becomes simple and easy. The error by this assumption will be discussed next.

Stuart ${ }^{[?]}$ showed that when a yarn having bending rigidity $B$ is in point contact with a rod having radius of $D / 2$, as shown in Fig. 11, the approximate relation between holding forces $P_{I}$ and $P_{I I}$ is given by

$$
\begin{gathered}
P_{\mathrm{I}} / P_{\mathbf{I}}=e^{\mu \theta^{\prime}}\left\{1-\frac{\mu\left(1+\mu^{2}\right)}{3 !}\left(\frac{1}{2} \Phi^{\prime}\right)^{3}\right. \\
\left.+\frac{\mu\left(1+\mu^{2}\right)\left(1+9 \mu^{2}\right)}{5 !}\left(\frac{1}{2} \Phi^{\prime}\right)^{5}+\cdots \cdots\right\}^{2} \cdots
\end{gathered}
$$

where $\mu$ is the coefficient of friction and $\Phi^{\prime}$ is the angular displacement of yarn between the points of application of the forces as shown in the figure.

This equation means that $P_{I} / P_{I I}$ is a function of $\mu$ and $\Phi^{\prime}$, and has no dependency on the bending rigidity $B$.

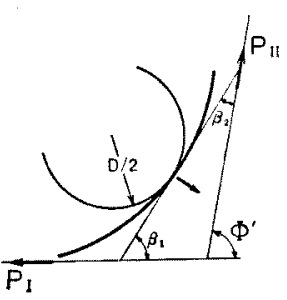

(a)

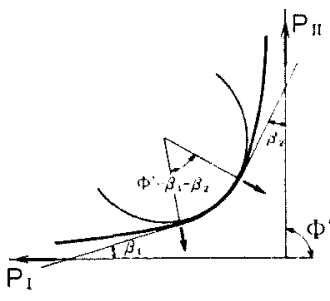

(b)
Fig. 11 Point contact (a) and line contact (b) 
If $\Phi^{\prime}=\pi / 2$ and $\mu=0.3$, the difference between two values of $\boldsymbol{P}_{I}$ is about $5 \%$ of their values, when one value is calclated by eq. (63) for point contact and another is for line contact with contact angle $\pi / 2$. In the structural model presented in Fig. 10, the difference becomes large, and yet only $10 \%$.

If both $P_{I}$ and $P_{I I}$ are large or either the bending rigidity of yarn or the curvature of the rod is small, line contact may arise. Then, using the symbols as shown in Fig. 11 (b), $P_{I} / P_{I I}$ is given by the following equation ${ }^{[7]}$,

$$
P_{1} / P_{I}=e^{* *}\left\{1-\frac{1}{2}\left(1-\mu^{2}\right)\left(\beta_{2}{ }^{2}-\beta_{1}^{2}\right)\right\} \cdots \cdots
$$

The correction due to the standard capstan equation is of the second order. Therefore. if the friction at the contact line is governed by Amonton's law, the correction by the capstan equation for yarn rigidity is usually small. So the error in this paper caused by the change of the contact angle in crossover region is also small.

In actual case, as yarns are compressed and deformed by lateral force, the contact angle $\Phi$ approachs $\Phi^{\prime}$ and the approximation error becomes negligible. Therefore, the assumption that the contact angle in corossover region is constant (here it is assumed to be $\pi$ ) does not cause serious error in this theory.

B When yarn slippage occurs in crossover region

$P$ and $Q$ can be calculated for each arc in unit structure. When the ratio of two $P_{s}$ on two arcs connected at the crossover point becomes larger than the value of $e^{\mu \pi}$, for example,

$$
P_{1 f} / P_{3 f}>e^{\mu \pi} \text { or } P_{3 f} / P_{1 f}>e^{\mu \pi} \ldots \ldots \ldots \ldots \ldots(65)
$$

then the slippage will occur on the crossover point. In this case, $P$ must be recalculated considering the frictional force as follows ${ }^{[4,5]}$. Firstly, $L_{a 1}$ and $L_{a 3 f}$ which satisfy the following equation must be calculated,

$$
P_{1 \delta} / P_{3 f}=e^{\mu \pi} \quad \text { or } \quad P_{3 f} / P_{1 f}=e^{r \pi \ldots \ldots \ldots \ldots \ldots}(66)
$$

Then, $P_{1 f}$ and $P_{3 f}$ are obtained.

\subsection{Calculation of $S$ and $F$.}

The shear force, $S$, acting on a unit structure is obtained as the sum of force components in the shear direction, and the tensile force, $F_{1}$, is as the sum of force components along the $\mathrm{X}_{1}$-axis.

In the case where the front-bar yarn takes type I deformation shown in Fig. 9, forces $F_{1}{ }^{\prime}, F_{1}{ }^{\prime \prime}, F_{2}{ }^{\prime}$ and $F_{2}{ }^{\prime \prime}$ (see Fig. 2) acting on the crossing point are calculated by

$$
\begin{aligned}
& F_{1}^{\prime}=R_{1,} \sin \left(\varphi_{0,1}-\left(\prime_{1 f}\right) \cos \alpha_{1 j}+R_{2} \cdot \sin \left(\varphi_{02} \prime\right.\right. \\
& +\left(a_{2} b\right) \cos \alpha_{2 b}+R_{3 b} \sin \left(5_{03} b+1 b_{3 b}\right) \cos \alpha_{3 b}
\end{aligned}
$$

$$
\begin{aligned}
& F_{1}^{\prime \prime}=R_{1 b} \sin \left(\varphi_{01 b} b+\omega_{1 b}\right) \cos \alpha_{1 b}+R_{2, r} \sin \left(\varphi_{02}\right. \\
& \text { (1)2f) } \cos \alpha_{2 f}+R_{3 f} \sin \left(\zeta_{03}+(1)_{3}\right) \cdots \cdots(68) \\
& F_{2}^{\prime}=R_{1} f \cos \left(\varphi_{01} f-\left(\omega_{1 j}\right)-R_{2 b} \cos \left(\varphi_{02} b+\omega_{2 i}\right)\right. \\
& +R_{3 b} \cos \left(\varphi_{03 b}-\omega_{3 b}\right) \\
& F_{2}{ }^{\prime \prime}=-R_{10} \cos \left(5_{010}+\omega_{10}\right)+R_{2 j} \cos \left(\varphi_{02} f-\omega_{2} j\right) \\
& -R_{3} f \cos \left(\varphi_{03} f+\omega_{3}\right) \text {. }
\end{aligned}
$$

where $\alpha$ is given by $\tan -1\left(\overline{O X_{3}} / \overline{O X_{1}}\right)$.

When the front-bar yarn takes type II deformation, suffixes $f$ and $b$ must be interchanged in eqs. (67) to (70).

Then, $F_{1}^{\prime}, F_{1}^{\prime \prime}, F_{2}^{\prime}$ and $F_{2}^{\prime \prime}$ obtained by eqs. (67) to (70) are substituted into eq. (3), and $S$ and $F_{1}$, can be calculated. The tensile force $\mathrm{X}_{2}$-axis per one course, $F_{2}$, is calculated by

$$
F_{2}=\left(F_{2}{ }^{\prime}+F_{2}^{\prime \prime}\right)\left(n_{f}+n_{t}\right) / 2
$$

because the fabric structure is assumed to be the layer of $\left(n_{f}+n_{b}\right)$ sheets having each a unit structure, as was already explained in the biaxial tensile theory. ${ }^{[4,5]}$

$$
\left.\begin{array}{l}
S(I)=S(\text { II }) \\
F(I)=F(\text { II })
\end{array}\right\}
$$

As above-mentioned, every course of the structure is subjected to two types of deformation, types I and II, as shown in Fig. 9. Therefore, even if shear or tensile force applied on each unit structure is the same for every course, there is a little difference between forces acting on two unit structures. For instance, when the front-bar yarn is subjected to type I deformation, the back-bar yarn is to type II deformation. In the actual deformation, the difference may be reduced by yarn slippage at the crossover point. The exact calculation under such slippage is too complicate to solve. For this reason, in this paper, the shear and tensile forces in these two cases were their average values respectively.

\section{Conclusion}

The shear deformation property of two-bar tricot fabrics has been analyzed, and the theory for calculating this property has been developed by using a structural model. This theory covers the general shear deformation of the fabrics including simple shear deformation. The theory makes the forces for deforming the fabrics calculable from the forces required for straightening the arcs, while the frictional effect between yarns is taken into consideration, but the elongation and compressive properties of yarns are out of consideration. The effect of them on the fabric property will be discussed in the next paper.

Grateful acknowlegment is made to members of Fiber Assembly Study Group of Department of Polymer Chemistry of Kyoto University for their useful discussions, and to Mr. K. Nakagawa for his helpful cooperation.

Journal of The Textile Machinery Society of Japan 


\section{Literature cited}

[1] P. Grosberg, B. J. Park; Text. Res. J., 36, 420 (1966)

[2] M. Niwa, S. Kawabata, H. Kawai; J. Soc. Fib. Sci. Tech, Japan, 26, 461 (1970)

[3] M. Niwa, S. Kawabata, H. Kawai; J. Soc. Fib. Sci. Tech, Japan, 26, 507 (1970)
[4] Y. Yanagawa, S. Kawabata, H. Kawai; J. Text. Mach. Soc. Japan, Eng. ed., 17, 15 (1971)

[5] Y. Yanagawa, S. Kawabata, H. Kawai; J. Text. Mach. Soc. Japan, Eng. ed., 18, 55 (1972)

[6] S. Kawabata; J. Text. Mach. Soc. Japan, 23, T30 (1970)

[7] I. M. Stuart; Brit. J. Appl. Phys., 12, 559 (1961) 